\title{
Towards Enabling Multihop Wireless Local Area Networks for Disaster Communications
}

\author{
Muhammad Bux Laghari, ${ }^{1}$ Hamayoun Shahwani ${ }^{D},{ }^{1}$ Syed Attique Shah ${ }^{1}{ }^{1}$ \\ Raja Asif Wagan $\mathbb{D}^{1},{ }^{1}$ Zahid Rauf, ${ }^{2}$ Ihsan Ali $\mathbb{D}^{3},{ }^{3}$ Sultan S. Alshamrani $\mathbb{D}^{4}$, \\ and Jaroslav Frnda $\left.{ }^{5}\right)^{5}$ \\ ${ }^{1}$ Faculty of Information and Communication Technology (FICT), Balochistan University of Information Technology, \\ Engineering and Management Sciences, Quetta, Pakistan \\ ${ }^{2}$ Dean Graduate Studies, Balochistan University of Information Technology, Engineering and Management Sciences, \\ Quetta, Pakistan \\ ${ }^{3}$ Department of Computer System and Technology, Faculty of Computer Science and Information Technology, University of Malaya, \\ 50603 Kuala Lumpur, Malaysia \\ ${ }^{4}$ Department of Information Technology, College of Computer and Information Technology, Taif University, P.O. Box 11099, \\ Taif 21944, Saudi Arabia \\ ${ }^{5}$ Department of Quantitative Methods and Economic Informatics, Faculty of Operation and Economics of Transport \\ and Communications, University of Zilina, 01026 Zilina, Slovakia
}

Correspondence should be addressed to Ihsan Ali; ihsanalichd@siswa.um.edu.my

Received 27 February 2021; Revised 12 April 2021; Accepted 23 April 2021; Published 19 May 2021

Academic Editor: K. Shankar

Copyright (C) 2021 Muhammad Bux Laghari et al. This is an open access article distributed under the Creative Commons Attribution License, which permits unrestricted use, distribution, and reproduction in any medium, provided the original work is properly cited.

xCalamities such as earthquakes and tsunami affect communication services by devastating the communication network and electrical infrastructure. Multihop relay networks can be deployed to restore the communication environment quickly in catastrophe-stricken areas. However, performance in terms of throughput is affected by deploying the relay networks. In wireless local area networks (WLANs), the primary purpose of multiband transmission employing multihop relay networks is to increase the throughput and reduce the latency. In the future, wireless networks are believed to carry high throughput, more data rates, and less latency by expanding bandwidth-demanding applications. Simultaneous multiband transmission in WLAN systems is considered to increase the coverage area without power escalation. Due to the inherent characteristics of different bands and channel conditions, transmission rates tend to be different. The impact of such conditions may cater to the disproportional distribution of data among bands, causing some of the bands to be overwhelmed, which incurs buffer overflow and packet loss. In contrast, the channel capacity of some of the bands remains underutilized. In this paper, we consider the channel conditions and transmission rates of each band on either side of the relay to address the problems mentioned above. Furthermore, this paper proposes a load distribution-based end-to-end traffic scheduling technique to improve system performance. The simulation results demonstrate the effectiveness of our proposed method with maximizing throughput and minimizing end-to-end delay.

\section{Introduction}

We live in a world that is a suitable example of the electronic cottage, requiring more and more Internet access and demand for high bandwidth with an increasing population. Therefore, to meet this daunting challenge, we consider a wireless local area network (WLAN) system that operates on different bands and multiple hops employing relay nodes.

Rehabilitation services are quite difficult in military tactical or catastrophe-stricken areas, where the communication infrastructure either has been demolished or does not exist earlier $[1,2]$. The restoration of the communication 
environment and coverage area can easily be enhanced by deploying multihop relay networks. But on the other hand, it results in low performance pertaining to throughput and latency due to the relay nature. Therefore, we require WLAN systems to raise the performance such as capacity, throughput, and end-to-end delay by relaying data over multiple hops. Particularly, relay transmission technology which transmits information over multiple devices from the source to destination nodes has gained a lot of attention for Device-to-Device (D2D) communication. Currently, WLAN with multiband frequencies is utilized to increase spectral efficiency. Despite the capabilities of WLAN systems to enhance the spectral resources by using license-free bands, WLAN yields higher power consumption with the increase of coverage area. However, it is the critical solution for WLAN communication to meet the increasing demands of wireless communication [3]. Figure 1 illustrates the restoration of communication with available WLAN deployments after disasters.

The Movable and Deployable Resource Unit (MDRU) has been stationed quickly in affected areas during a massive earthquake and tsunami in Japan on March 11, 2011. Each MDRU is a container transported by helicopters in disaster zones, consisting of routers, switches, power distribution units, and other computing equipment to provide real-time communications [4]. The MDRU was designed to accommodate 5000 users, whereas every cell consists of 18 access points. Regardless of potentials, MDRU has the ability to restore communication in a brief span of time.

Dense deployment of WLAN decreases spectral resources because of the higher demand for machine-to-machine communication and the Internet of things. So, spectral efficiency can be improved by using unlicensed bands. On the other hand, the single-band communication over multihop increases coverage area and enhances latency due to relaybased communication. Nevertheless, the WLAN system uses multiple unlicensed bands such as $980 \mathrm{MHz}, 2.4 \mathrm{GHz}$, and $5 \mathrm{GHz}$ to increase spectral resources while taking into consideration multiband relay communication.

Performance is a great concern in one-hop WLANs, with increasing bandwidth-hungry applications. The compound annual growth rate of WLAN traffic between 2016 and 2021 is 47 percent [5], as shown in Figure 2, which means 1000 times more bandwidth is required as compared to the current capacity level in the next few decades.

With the increasing number of bandwidth-intensive applications, it is expected that future wireless networks will put up a persistent rise in demand for efficient spectrum utilization, high data rates, low latency, and better throughput. In fact, as the advantages of wireless connectivity go beyond smartphones and tablets, wireless services would require additional utility devices. Globally, the average number of devices per capita is 7.83 [6], and their distribution is shown in Figure 3.

Numerous specialized methods and techniques have been implemented to satisfy this formidable demand in the current technological developments, such as bandwidth aggregation, spatial multiplexing, massive multiple-input multiple-output (MIMO), and dense deployment of the devices [7]. Nonetheless, the efficiency anticipated from next-generation wireless networks, such as supplying orders

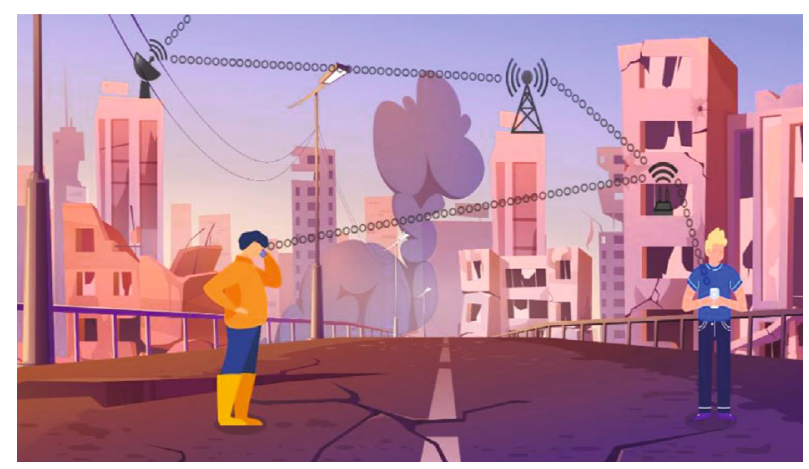

FIgURE 1: Restoration of communication with available WLAN deployments after disasters.

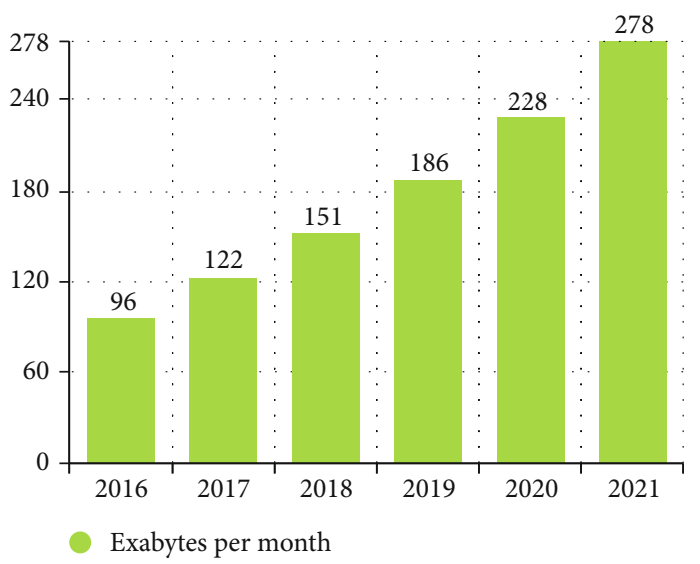

Figure 2: Annual traffic mobile growth.

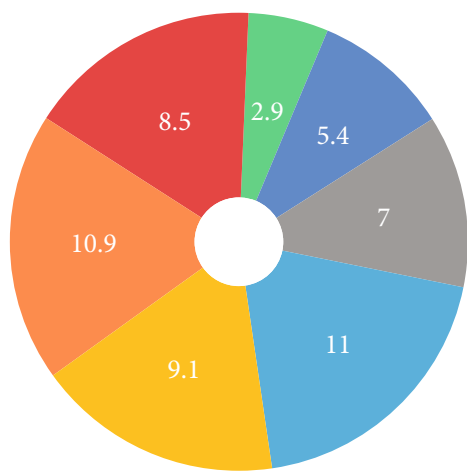

- Asia Pacific

- Middle East

- Latin America

- North America

- Central \& Eastern Europe

- Western Europe

- Global

Figure 3: Average number of devices per capita.

of magnitude with more capacity to meet the increasing bandwidth demand, is already overwhelming. To this end, we envision multiband WLANs, which is a promising 
solution to meet the bandwidth requirement of conventional WLAN users by increasing the relaying network's capacity. We envisage a system where nodes can operate simultaneously over multiple bands such as $5 \mathrm{GHz}, 2.4 \mathrm{GHz}$, and $980 \mathrm{MHz}$.

Generally, all the requirements are not being fulfilled by a single band. Single-band receiver works only with one frequency band, while multiband receiver fetched several frequencies. A multiband receiver is more reliable and ensures a higher coverage area than the single-band receiver. We need to consider the inherent characteristics of different frequency bands and channels from a most appropriate band in accordance with its performance. For instance, the $2.4 \mathrm{GHz}$ band is already congested with electronic devices because they hold only three orthogonal channels; therefore, they provide better device compatibility as compared to $5 \mathrm{GHz}$. The limited number of channels in addition to overcrowdings of the band led to surge interferences from nearby devices and intermittent connectivity problems that outcomes less throughput. The $5 \mathrm{GHz}$ provides less room for cochannel interference, comparatively to the $2.4 \mathrm{GHz}$. Similarly, the $2.4 \mathrm{GHz}$ is less affected by path loss comparatively to $5 \mathrm{GHz}$ while providing a more significant coverage zone than $5 \mathrm{GHz}$. In contrast, $5 \mathrm{GHz}$ is not as crowded as it has 12 orthogonal channels [8]. Furthermore, channel bonding space in $5 \mathrm{GHz}$ is more than the $2.4 \mathrm{GHz}$, and thereby channel width can be increased from $20 \mathrm{MHz}$ to $80 \mathrm{MHz}$ as per requirement in $5 \mathrm{GHz}$ [9]. The $2.4 \mathrm{GHz}$ band will support speed between $450 \mathrm{Mbps}$ and $600 \mathrm{Mbps}$, while $5 \mathrm{GHz}$ will support speed up to $1300 \mathrm{Mbps}$. Thus, if the node is in a point-to-point connection with AP, a channel assignment from $5 \mathrm{GHz}$ would be preferable as per the signal-to-noise ratio (SNR) condition. Yet, the spectral efficiency needs to be improved, gratitude to wireless communication, which impels operators to reduce their spectrum utilization by employing multiple bands.

The proposed system allows synchronous use of all three bands to send and receive the data to maximize the throughput. We believe that simultaneous transmission across multiple bands will prove a productive way to meet future network traffic demands, keeping in mind that different bands have different responses to physical conditions, such as distance and interference. With the provision of multiple bands to each node, we can experience the different bands with their basic characteristics, by this means benefiting with better radio frequency (RF) spectrum simultaneously. However, wireless network performance is limited by multiple hops [10]; it will provide multi-Gbps throughput to end-users in a cost-effective way. Transmission over multiple bands will serve as a dominant proposal to establish future wireless applications.

In a single-hop network, the source node and destination node are directly connected to one another. In the two-hop relaying link, the physical channel between the source and the relay is called the relay link, whereas between the relay and the destination it is called the access link. However, the relay node is used between the source node and the destination node in the multihop WLAN system to support transmission [11]. Multihop networks are deployed in seminars, campuses, exhibitions, and libraries to increase the coverage area. These networks can be deployed in disaster-hit areas to provide rehabilitation and relief services to affected victims by establishing fast communication infrastructure.

However, certain issues need to be overcome before realizing the actual benefits of multiband WLANs. The performance, such as throughput and latency in multihop multiband WLAN systems, is affected by various factors such as link condition at pre- and postrelay links, packet loss, buffer overflow, channel underutilization, and packet reordering delay. In contrast, channel conditions such as fading and propagation loss vary in each band in multiband relaying communication. This results in a discrepancy within the data rates between reception and transmission at the relay node. When a source-relay link is more significant than the relaydestination link, it causes data loss from the buffer overflow; however, if the source-relay link is less than the relaydestination link, it results in a particular channel having more capacity. The relay nodes communicate, and the relay node or the link after the relay node cannot accommodate the incoming traffic, which causes channel underutilization. Packet reordering is the important matrix that must be taken into account while considering wireless networks' performance, where the receiving order of packets differs from the sending order. Similarly, packets that traverse multiple hops and the frequency of traffic switching from one band to another affect end-to-end delays.

In this research, initially, the performance estimation of every band will be performed; later on, we formulate mixed-integer linear programming (MILP). Moreover, we design a load distribution-based end-to-end traffic scheduling technique that decreases latency and increases throughput. The MILP will be designed for single-hop, directly connected users. Next, we develop an extension by incorporating the relay nodes. Considering the performance of the links, the proposed technique will distribute the incoming traffic among the bands so that their capacities are optimally utilized, while load over bands will be varied in such a way that delays of all the bands are made equal, thereby completely avoiding packet reordering at the receiver's end.

In this research work, our main technical contributions are as follows.

(i) We outline the factors that affect the delay and throughput in multiband WLANs, and based on those factors, the MILP is formulated

(ii) Considering MILP formulation, we propose a novel load distribution-based end-to-end traffic scheduling technique to improve the system performance

(iii) The simulation results validate the effectiveness of our proposed technique by maximizing throughput and minimizing end-to-end delay

The rest of the paper is organized as follows. We study the optimization and performance analysis of different wireless network schemes in the literature review in Section 2. We illustrate our system model and problem formulation in Section 3. The load distribution-based end-to-end traffic 
scheduling algorithm to minimize latency and maximize throughput is discussed in Section 4. The performance evaluation is given in Section 5. The results and discussions are proposed in Section 6. Finally, in Section 7, we outline the conclusion and future work.

\section{Literature Review}

Multiband WLAN is a promising solution guaranteed to enhance the multihop relay networks and satisfy the bandwidth need of conventional WLAN users. Manipulating the attributive nature of different bands will give rise to the creative performance of these networks. Capacity enhancement via channel switching within a band has been studied thoroughly in [12]. To date, many researchers have considered the idea of mobile networks and multiband multihop transmissions in WLANs. The band switching mechanism in cellular networks is proposed in [13]. In the proposed architecture, when a primary node differs the band, all the secondary nodes alter their bands, respectively. The technique for selecting a suitable band is yet needed in this research, for that scenario framework is unable to support multiband networks.

Nevertheless, the $2.4 \mathrm{GHz}$ band is already congested with electronic devices because they hold only three orthogonal channels [14]; therefore, they provide better device compatibility as compared to $5 \mathrm{GHz}$ but suffer higher cochannel interference. On the other hand, $5 \mathrm{GHz}$ supports a higher speed than $2.4 \mathrm{GHz}$ but provides less coverage area than $2.4 \mathrm{GHz}$ [15]. Packet reordering does not occur more than $3 \%$ in a single path, whereas it exceeds $30 \%$ in multipath propagation [16]. Packet reordering is less in a single path, whereas it is higher on multipath, because of routing, flapping, and retransmission on TCP/UDP and causes congestion on the network. The authors in [16] proposed a method to reduce delay and packet reordering delay by $40 \%$ for both CBR and VBR applications.

Low latency is a crucial demand to improve networks' communication performance in different scenarios such as security and emergency. The authors in [17] proposed an information theoretic-based approach to compute the cooperative strategies for each relay between amplify and forward (AF) and decode and forward (DF). The error exponent identifies the trade-off between reliability and code block (latency). Such trade-off permits high error probability tolerance at the weaker channels. The increased SNR increases the transmission rate ultimately for all relay nodes to perform AF. However, the reduced SNR decreases the transmission rate eventually for all relay nodes to perform AF and surges delay at each hop as compared to DF, albeit the AF node increases larger delay than DF at longer distances.

The multiband could use any available channel perceived by multiband sensing on other bands, in case of the channel on any band is busy, whereas legacy terminals fail to obtain them. Consequently, the multiband WLAN uses idle spectral resources through various bands; hence, it caters to minimize delay by improving spectral efficiency. With all these approaches, regardless of the channel's conditions, the band

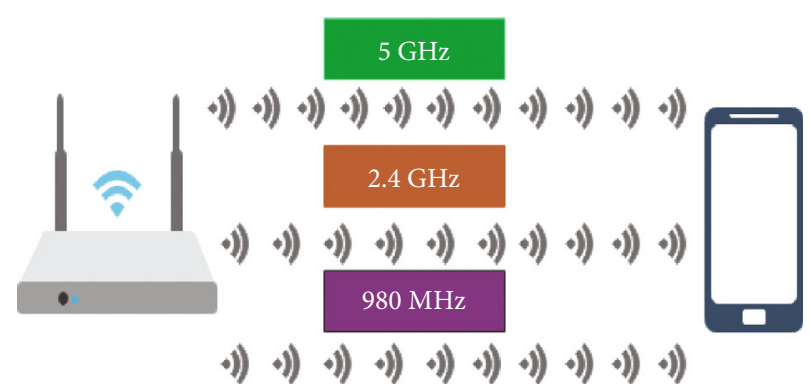

FIGURE 4: Simultaneous transmission.

is always changed to produce an efficient spectrum. This causes a critical delay that adversely impacts the latency and throughput, whereas the contribution of band switching delay is not minor [18]. Bitrate on source-relay link can be dissimilar to relay-destination link and hence results in the buffer overflow, and data frames can be lost. To fill these research gaps, we envision multiband multihop relay networks that simultaneously transmit over multiple bands, such as $5 \mathrm{GHz}, 2.4 \mathrm{GHz}$, and $980 \mathrm{MHz}$, as shown in Figure 4, depicting an alternative to switching between the bands.

In [19-21], the authors extended and supplemented the previous studies with an adaptive channel selection method to reduce the transmission delay. In contrast, a channel with higher SNR is selected as per availability in the initial attempt rather than lowering the bitrate. Else, bitrate is selected in the following attempt. The Decode-and-Forward (DF) scheme increases the coverage area without expanding the source node's transmission power [19]. Nevertheless, sending and relay transmission processing in the relay node caters to enhance the latency. Furthermore, Truncated Decode and Forward (TDF) is a substantial solution to cut down the imperative latency complication by obtaining bit sequence from relay transmission. However, the TDF technique surges total latency due to retransmission. In addition to that, decoding performance deteriorates as the frame error increases [20].

Moreover, Adaptive Relay Transmission Selection (ARTS) scheme has been employed for channel selection to evaluate an anticipated latency using the frame error rate (FER) estimation acquired from SNR. ARTS technique selects relay transmission with minimum latency [21], whereas ARTS scheme is more affected by overhead using relay transmission as compared to the TDF scheme.

In limited delay communication, information is being transmitted from source to destination through reliability (error probability). DF and AF schemes are compared with a point-to-point scheme to estimate the end-to-end delay analysis, pertaining to SNR [22]. Basically, DF not only ensures reliability requirements at the destination but at each relay node. In contrast, AF performs decoding only at the destination to achieve a reliability requirement. The authors investigated sufficient conditions at which DF and AF show less latency than the P2P technique by achieving a high transmission rate at higher SNR. The 
DF attains less latency than P2P at the following condition with high SNR.

$$
M_{h}\left(\mathrm{SNR}_{1}\left|\mathrm{~dB}, \mathrm{SNR}_{2}\right| \mathrm{dB}\right)>2\left(\mathrm{SNR}_{0} \mid \mathrm{dB}\right) .
$$

The AF achieves less latency than P2P at the below condition with high SNR.

$$
\frac{M_{h}\left(\mathrm{SNR}_{1}, \mathrm{SNR}_{2}\right)}{2} \mid \mathrm{dB}>2\left(\mathrm{SNR}_{0} \mid \mathrm{dB}\right) .
$$

The authors in [23] presented a comparative analysis for multihop relay communication between $\mathrm{P} 2 \mathrm{P}, \mathrm{DF}$, and $\mathrm{AF}$ methods. The DF scheme ensures less latency than P2P at higher SNR as follows:

$$
\frac{1}{\log _{2}\left(\mathrm{SNR}_{\mathrm{DF} 1}\right)}+\frac{1}{\log _{2}\left(\mathrm{SNR}_{\mathrm{DF} 2}\right)}<\frac{1}{\log _{2}\left(\mathrm{SNR}_{\mathrm{P} 2 \mathrm{P}}\right)} .
$$

However, AF has lower latency than P2P at higher SNR as below

$$
\frac{2}{\log _{2}\left(\mathrm{SNR}_{\mathrm{AF}}\right)}<\frac{1}{\log _{2}\left(\mathrm{SNR}_{\mathrm{P} 2 \mathrm{P}}\right)}
$$

While considering more number of hops, DF performs better than AF, because at each hop, latency increases by amplifying noise signals. In the proposed condition, latency is subject to SNR. If the relays are properly set up between source and destination, it reduces latency as compared to the P2P approach.

In [24], the authors investigated the min-max optimization problem to maximize the capacity bounds by utilizing optimal power and bandwidth allocation strategies. Compared to channel conditions, joint optimization methods yield higher transmission rates than a single power optimization scheme. The resources are allocated in order to achieve high transmission rates under a given channel condition. However, channel capacity enhancement has not been discussed in this study.

The utilization of cooperative communication (CC) multihop networks proves better results than existing single-hop wireless networks [25]. Mathematical optimization techniques, namely, branch and cut, improve rate gains significantly. The CC with DF and AF has been investigated to analyze the communication performance of the relay node while P2P without relay node. Nevertheless, the feasible solution construction (FSC) algorithm addresses the solution process in three steps, namely, path determination, CR assignment, and flow recalculation.

The MILP performs better for small and medium-scale networks, whereas it does not provide optimal solutions for large-scale networks. To cater to that, the authors in [26] proposed AP coverage adjustment problem (ACAP) and user association optimization problem (UAOP). When the AP is overcrowded, it pressures few users to switch APs, by turning down its transmission power to reduce its coverage area. In case the AP is lightly loaded, it attracts some users towards APs, by increasing its transmission power to expand its coverage area. The joint ACAP and UAOP attain better performance and provide optimal solutions for large-scale networks quickly with low overhead. However, the gap between AP utilization increases by increasing traffic demands. Additionally, maximum AP utilization remains still stable while varying the number of APs, which increases the load on concerned APs, thereby ignoring others.

Minimum length scheduling problem has been investigated in multiradio multichannel multihop cognitive cellular radio $\left(\mathrm{M}^{3} \mathrm{C}^{2} \mathrm{~N}\right)$ to enhance the capacity and throughput of cellular networks using hybrid mode. With hybrid mode, nodes close to a base station communicate with the base station directly in one hop. The other nodes farther away needed to communicate via multiple hops, i.e., in ad hoc mode. The $\epsilon$-bounded approximation algorithm has been examined to achieve both upper and lower bounds tight solutions quickly without finding maximal independent sets. However, $0 \leq \epsilon<1$ is still system control parameter [27].

In [28], the authors designed a network management centre to address the critical challenges and investigate traffic load balancing problems among the gateways in wireless mesh networks (WMN). Two perspectives are suggested: firstly, a balanced distribution of bandwidth to be allocated per user; secondly, consider users' bandwidth demands dynamically from a disaster zone. The authors examined a useful fairness index per user with proposed approaches, average hop count, and a smaller number of MR handover events, approximately to 40, which exceeds 200 in former techniques. On the other hand, the average hop count is increased in proposed methods compared to the HWMP method.

The mobile stations change their locations in real time, which leads to unbalanced load distribution among APs. The load imbalance degrades the WLAN performance. The authors in [29] proposed a load balancing algorithm to improve network performance. The better performance is achieved with the proposed load balancing algorithm with reference to network throughput and data dropped in WLAN. However, association and reassociation of load with access points increase the switching delay, hence ultimately enhancing the time complexity of the proposed algorithm.

Satellite communication is an infallible option for disaster management and emergency relief. High-throughput satellite is an alternate candidate as compared to terrestrial networks, which address resilient satellite networks by selecting an optimum gateway based on the traffic demand at neighboring user-link beams [30]. The authors proposed a frequency allocation scheme to improve the utilization of both user-link and feeder-link bandwidth by selecting optimal gateways. Hence, it reduces the number of operational gateways comparatively to the fixed allocation method to achieve the system's throughput as per requirement. However, the digital channelizer receives signals at the same port from terminals and gateways; therefore, interchannel interference caused by filter bank degrades the SINR due to the imbalance received power.

Green multiband channel selection of portable devices using IEEE 802.11 ad has been conducted in $[31,32]$. In addition, authors addressed the issues and challenges of the 
TABLE 1: Comparative analysis of various existing schemes.

\begin{tabular}{|c|c|c|}
\hline Technique & Advantages & Limitations \\
\hline Decode-and-Forward (DF) [16] & $\begin{array}{l}\text { Increases coverage area without expanding } \\
\text { the transmission power of source node }\end{array}$ & $\begin{array}{c}\text { Relaying transmission caters to enhance } \\
\text { the latency }\end{array}$ \\
\hline Truncated Decode and Forward (TDF) [17] & $\begin{array}{l}\text { Improves spectral efficiency while } \\
\text { minimizing delay }\end{array}$ & Increases total latency due to retransmission \\
\hline $\begin{array}{l}\text { Adaptive Relay Transmission Selection } \\
\text { (ARTS) [18] }\end{array}$ & $\begin{array}{l}\text { Selects relay transmission with } \\
\text { minimum latency }\end{array}$ & $\begin{array}{l}\text { More affected by overhead with } \\
\text { relay transmission }\end{array}$ \\
\hline Information theoretic approach [19] & $\begin{array}{l}\text { If relay channels are strong and robust, then } \\
\qquad \mathrm{AF} \text { and DF attain less latency }\end{array}$ & $\begin{array}{c}\text { If uplink and downlink are weak channels, } \\
\text { then AF and DF cater negative impact } \\
\text { on latency }\end{array}$ \\
\hline Information theoretic technique [20] & $\begin{array}{l}\text { While considering more number of hops, DF } \\
\text { performs better than AF }\end{array}$ & $\begin{array}{c}\text { At each hop, latency increases by amplifying } \\
\text { noise signals }\end{array}$ \\
\hline $\begin{array}{l}\text { Joint power and bandwidth allocation } \\
\text { strategies [21] }\end{array}$ & $\begin{array}{l}\text { Particular to channel conditions, joint } \\
\text { optimization methods yield higher } \\
\text { transmission rates as compared to single } \\
\text { power optimization scheme }\end{array}$ & $\begin{array}{c}\text { Channel capacity enhancement has not been } \\
\text { discussed in these studies }\end{array}$ \\
\hline $\begin{array}{l}\text { Feasible solution construction (FSC) } \\
\text { algorithm [22] }\end{array}$ & $\begin{array}{c}\text { Joint optimization problem of flow routing } \\
\text { and relay node assignment for concurrent } \\
\text { sessions to improve capacity of } \\
\text { wireless networks }\end{array}$ & $\begin{array}{l}\text { It is hard to solve the computational } \\
\text { complexity of this algorithm }\end{array}$ \\
\hline $\begin{array}{l}\text { AP coverage adjustment problem (ACAP) } \\
\text { and user association optimization problem } \\
(\mathrm{UAOP})[23]\end{array}$ & $\begin{array}{l}\text { Provides optimal solutions for large-scale } \\
\text { networks quickly with low overhead }\end{array}$ & $\begin{array}{l}\text { Gap between AP utilization increases by } \\
\text { increasing the traffic demands }\end{array}$ \\
\hline$\epsilon$-bounded approximation [24] & $\begin{array}{c}\text { Executes both upper and lower bound tight } \\
\text { solutions quickly without finding maximal } \\
\text { independent sets }\end{array}$ & $\begin{array}{l}\text { However, } 0 \leq \epsilon<1 \text { is still system } \\
\text { control parameter }\end{array}$ \\
\hline Heuristic-based approach [25] & $\begin{array}{c}\text { Examined good fairness index per user, } \\
\text { average hop count, and less number of MR } \\
\text { handover events }\end{array}$ & $\begin{array}{l}\text { The average hop count is increased as } \\
\text { compared to HWMP method }\end{array}$ \\
\hline Load balancing algorithm [26] & $\begin{array}{c}\text { Provides better performance with reference } \\
\text { to network throughput and data dropped } \\
\text { in WLAN }\end{array}$ & $\begin{array}{l}\text { Association and reassociation of load with } \\
\text { access points increase the switching delay }\end{array}$ \\
\hline Frequency allocation scheme [27] & $\begin{array}{l}\text { Reduces the number of operational gateways } \\
\text { comparatively to the fixed allocation method }\end{array}$ & $\begin{array}{l}\text { Digital channelizer degrades the SINR due to } \\
\text { the imbalance received power }\end{array}$ \\
\hline
\end{tabular}

multiband multichannel selection in WLAN. Table 1 shows the comparative analysis of various existing schemes.

\section{System Model}

Our proposed system forms a combination of the bands, where all the three bands are utilized simultaneously to transfer the load. The proposed multihop relay-based WLAN system operates on three different bands, namely, $980 \mathrm{MHz}$, $2.4 \mathrm{GHz}$, and $5 \mathrm{GHz}$. The source node's data frames are divided into three segments within three bands on the links, depending upon individual bands' performance on outgoing links. Each segment of frames is forwarded concurrently. After reaching the destination, the frames are rearranged and delivered to each segment for application after the required processing at sublayers as given in Figure 5.

3.1. Problem Formulation. We formulate the integer linear program (ILP) for the point-to-point connected sourcedestination pair. Afterward, multiband multihop relays are incorporated for the extension of the system model. MILP problems may contain objective function, constraints, and variables. Our primary function is to minimize the delay by improving system performance. We write the objective function as follows:

$$
\begin{aligned}
& \text { Minimize } D \\
& D=\frac{\text { Incoming Traffic }}{\text { Transmission Rate }} \\
& D_{i}=\frac{(\mathrm{IT})_{i}}{T_{i}}
\end{aligned}
$$

Subject to constraints.

3.2. Share Ratio Constraint. The sum of share ratio of all the bands should always be equal to 1 . Hence, to guarantee that the sum of all bands should not exceed the total link capacity,

$$
\sum_{i=1}^{N} k_{i}=1
$$




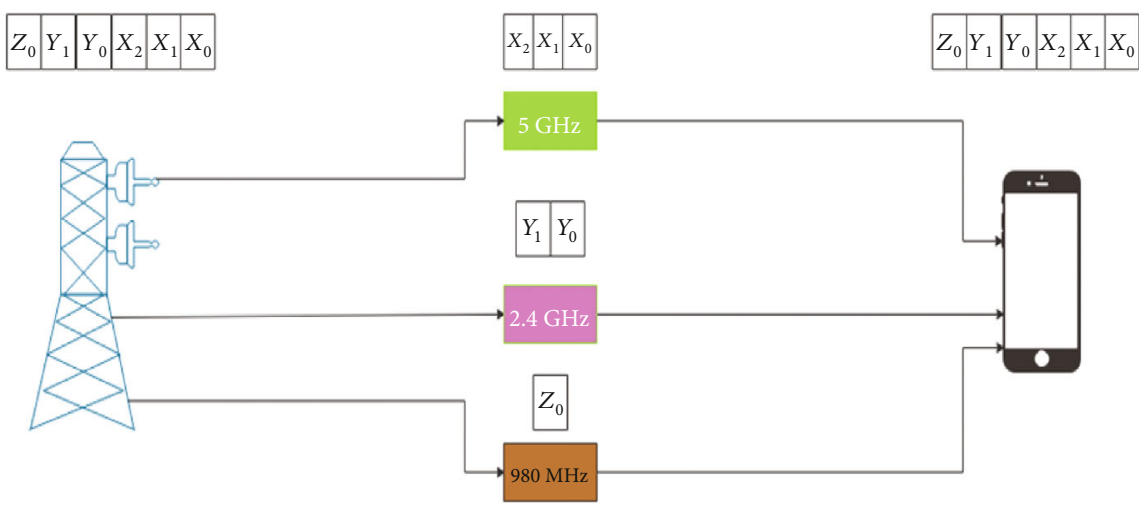

FIGURE 5: A simplified illustration model of multihop WLAN.

3.3. Load Share Constraint. Individual share cannot be zero in case of traffic load exists. Each band has to participate in order to improve the objective function.

$$
k_{i}>0 \forall L>0 \text {. }
$$

3.4. Capacity Constraint. The transmission rate must be positive invariably and cannot exceed the capacity of the band $i$. Basically, this is not possible to possess a negative transmission rate.

$$
0<T_{i} \leq C_{i}
$$

3.5. Delay Constraint. The delay of each band must be equal in order to ensure the smooth delivery of the data packets. The packets will arrive out of order, subject to unequal delay, consequently resulting in packet reordering delay.

$$
D_{i}=D_{j}
$$

3.6. Positive Share Ratio Constraint. Each band must ensure a positive share ratio of traffic load. Generally, for any band, it is not possible to have a negative load.

$$
k_{i} \geq 0
$$

3.7. Hop Count Constraint. Hop count is always equal to 1 for single-hop communication and at least 2 or greater than 2 for multihop transmission. This ensures at least single intermediate node between source and destination.

$$
H_{s, d} \geq 1
$$

3.8. Transmission Rate Constraint. The transmission rate of the faster band must be equal to the sum of the transmission rate $s$ of the remaining two bands in order to ensure equal end-to-end delay.

$$
T_{X}=T_{Y}+T_{Z}
$$

3.9. User Association Constraint. The number of users associated with band $Y$ should be less than users associated with band $X$ and greater than users associated with band $Z$.

$$
Z_{U}<Y_{U}<X_{U}
$$

3.10. Channel/Band Switching Constraint. The transmission delay of the new channel plus channel switching delay should be less than the transmission delay of the current channel. Frequently switching of the channel will degrade the throughput and enhance latency.

$$
\mathbb{d}_{t}^{n}+\Delta<\mathbb{d}_{t}^{c}
$$

We design MILP, which ensures all delays being equal implying that packets will arrive in proper format at the receiver's end, guaranteeing that no packet reordering delay will occur. However, variables can take nonintegral values such as delay, therefore formulating turnout to be MILP, whereas all the variables are independent of each other or simply they are disconnected; hence, MILP is NP hard, too. All MILP problems are nonpolynomial type, nondeterministic problems; thus, it is hard to find the computational complexity of all MILP problems; therefore, such problems are considered as NP-hard problems.

\section{Proposed Solution}

In this section, we propose a solution to the formulated problem using load distribution-based end-to-end traffic scheduling technique. The technique used in the proposed algorithm optimally assigns traffic load to the bands depending upon their performance in a way that all the constraints are resolved, and the end-to-end packet order is maintained, that is, all the packets arrive perfectly in order, thus totally avoiding traffic reordering delay. The outcome of the algorithm will share the load that the band will get when the total load is distributed among the coalition bands. The shared load is according to the performance of the individual band.

For Algorithm 1 analysis, suppose that the total incoming traffic load is $50 \mathrm{Mb}$. Nonetheless, three bands, namely, $X, Y$, and $Z$, have been assumed to address the problem. The 


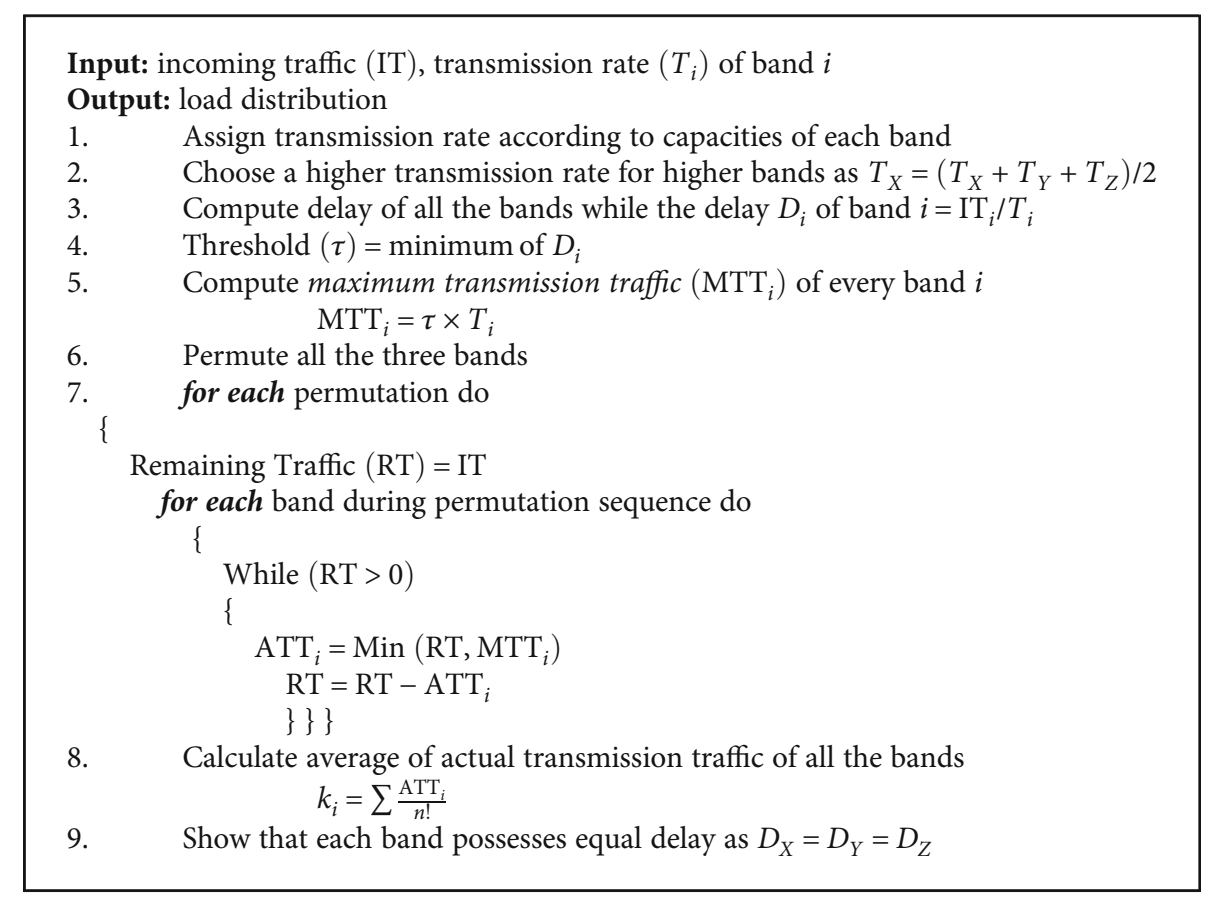

Algorithm 1: Load distribution-based end-to-end traffic scheduling technique.

transmission capacity for these three bands is $5 \mathrm{Mbps}$, $3 \mathrm{Mbps}$, and $2 \mathrm{Mbps}$, accordingly, as mentioned in Step 1. However, the faster band will hold a higher transmission rate as compared to others in Step 2. Our goal is to distribute the traffic among the three bands unequally in such a way that the constraints are satisfied and packet ordering is conserved. Using the delay equation shown in the MILP, the individual delays of the three bands will be calculated that comes out to be 10 seconds for band $X$, about 16.7 seconds for band $Y$, and 25 seconds for band $Z$ in Step 3 . However, band $X$ is the fastest and band $Z$ is the slowest among all. In Step 4, we have to choose our threshold value. In this case, the fastest band happens to have a delay of 10 seconds; therefore, we have chosen this value as a threshold value. In Step 5, we need to compute maximum transmission traffic $\left(\mathrm{MTT}_{i}\right)$ of all the three bands. Table 2 represents the detailed elucidation of maximum transmission traffic of each band.

Next, as per Step 6, we have to permute all three bands for the maximum possible permutation. Similarly, in Step 7, we compute $\mathrm{ATT}_{i}$ of all the bands as per permutation order defined in Table 3.

Next, as per Step 8, we calculate actual transmission traffic $\left(\mathrm{ATT}_{i}\right)$, with respect to row-by-row through all the rows and all the bands. We will take the average of all ATT's of all the bands. Upon taking the average of six rows for all the bands, it turned out that band $X$ will get $25 \mathrm{Mb}$ out of $50 \mathrm{Mb}$ while bands $Y$ and $Z$ will get $15 \mathrm{Mb}$ and $10 \mathrm{Mb}$, respectively. In the end, we formulate the delay balancing in Table 4.

\section{Performance Evaluation}

The topology used for performance evaluation is given in Figure 6. There is a source AP denoted by "S" and a relay
TABle 2: An elucidation of maximum transmission traffic of each band.

\begin{tabular}{lccc}
\hline Bands & $\tau$ & $T_{i}$ & $\mathrm{MTT}_{i}$ \\
\hline$X$ & 10 & 5 & 50 \\
$Y$ & 10 & 3 & 30 \\
$Z$ & 10 & 2 & 20 \\
\hline
\end{tabular}

TABle 3: An illustration of load sharing using the proposed technique.

\begin{tabular}{lccc}
\hline Bands & $X$ & $Y$ & $Z$ \\
\hline$X Y Z$ & 50 & 0 & 0 \\
$X Z Y$ & 50 & 0 & 0 \\
$Y X Z$ & 20 & 30 & 0 \\
$Y Z X$ & 0 & 30 & 20 \\
$Z X Y$ & 30 & 0 & 20 \\
$Z Y X$ & 0 & 30 & 20 \\
Load & 150 & 90 & 60 \\
\hline
\end{tabular}

TABle 4: Demonstration of equal delay with variable load.

\begin{tabular}{lccc}
\hline Bands & Load on each band & Capacity of each band & Delay \\
\hline$X$ & 25 & 5 & 5 seconds \\
$Y$ & 15 & 3 & 5 seconds \\
$Z$ & 10 & 2 & 5 seconds \\
\hline
\end{tabular}




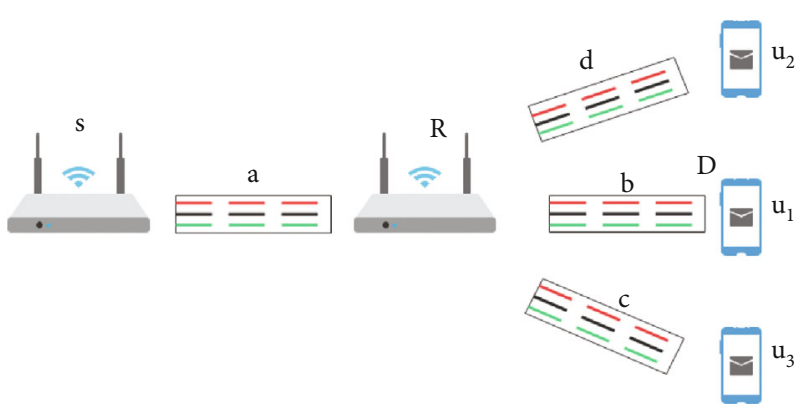

FIgURE 6: Topology used for performance evaluation.

AP labeled by "R," whereas several end-user nodes are indicated by " $u$." It is worth mentioning that as an illustration only a single relay is shown here; however, in reality, there can be any number of relay nodes. Initially, the single source and destination pair is used to represent the share fairness and load distribution. Afterwards, additional nodes can be used for relaying to support the transmission. Simply, a node $r$ (where $1<r<d$ ) receives a packet from $r-1$ th node and forwards it to $r+1$ th node before the frame reaches $d$ th node, which is generally a destination node.

In this research, SNR is obtained based on the distance between the access points (APs) and the distance between APs and end-nodes. For this purpose, we use QAM-64 modulation scheme and Single-Input-Single-Output (SISO) omnidirectional antenna. Having obtained noise, the SNR was attained by using the equation below.

$$
\mathrm{SNR}=\frac{\text { Signal }}{\text { Noise }}
$$

Thereafter, we have calculated bit error rate (BER) from SNR. BER for QAM-64 is given in the equation below.

$$
\mathrm{BER}=\frac{7}{12 Q(\sqrt{2 \partial})}
$$

Here, $\partial$ is the symbol level of SNR and $Q$ is the standard normal CDF. $Q$ can be calculated using the following relation.

$$
Q(\sigma)=\frac{1}{2}-\frac{1}{2} \operatorname{erf}\left(\frac{\sigma}{\sqrt{2}}\right)
$$

where $\sigma$ is the standard deviation and erf is the error function. Subsequently, after obtaining BER, we compute the packet error rate that will eventually give us the packet success rate. The packet error rate was determined using the equation below.

$$
\operatorname{PER}=1-(1-\mathrm{BER})^{l}
$$

TABLE 5: Parameters utilized.

\begin{tabular}{lc}
\hline Parameters & Value \\
\hline Radios per node & 3 at each node \\
Bands utilized & $5 \mathrm{GHz}, 2.4 \mathrm{GHz}$, and $980 \mathrm{MHz}$ \\
Bandwidth & \\
$5 \mathrm{GHz}$ & $20 \mathrm{MHz}$ \\
$2.4 \mathrm{GHz}$ & $20 \mathrm{MHz}$ \\
$980 \mathrm{MHz}$ & $1 \mathrm{MHz}$ \\
Background noise & $-90 \mathrm{dBm}$ \\
Transmission rate & As per SNR \\
Packet length & $512 \mathrm{bytes}$ \\
Channel switching delay & $130 \mu \mathrm{s}$ \\
Transmit power & $30 \mathrm{~dB}$ \\
Modulation technique & QAM-64 \\
Internode distance & $20 \mathrm{~m}$ \\
\hline
\end{tabular}

Here, $l$ is the packet length, and the value of which is given in Table 5. From this, the packet success rate was calculated as follows.

$$
\text { PSR }=1-\text { PER }
$$
follows.

Having obtained PSR, throughput can be determined as

$$
\text { Throughput }=T * \text { PSR. }
$$

\section{Simulation Analysis}

We have demonstrated our proposed method's appositeness with the help of the acquired results in this section. Referring to Figure 6, the performance between source node " $\mathrm{S}$ " and relay node " $R$ " has been experienced first, and then, the entire end-to-end results are shown. We have observed the losses, SNR, and transmission rate of the bands that have been used before embarking on the performance of our proposed technique. These results were obtained in the process of calculating the transmission rate for the different bands.

6.1. Signal-to-Noise Ratio with respect to Distance. Based on the obtained losses, we compute the signal-to-noise ratio of all the bands. Besides that, we also experience the $60 \mathrm{GHz}$ band's comparative results with the rest of the bands. In Figure 7, we observe the SNR of all the bands and their impact by changing distance. The higher frequencies because of more losses have lower SNR. SNR decreases with respect to distance. SNR of $980 \mathrm{MHz}$ is the highest among all the bands; $60 \mathrm{GHz}$ attains the lowest SNR.

6.2. Throughput Analysis. The bitrate giving the highest throughput is chosen as the optimal bitrate later on used for packet transmission. For bandwidth being the same, higher SNR results in a higher bitrate. The bandwidth for $2.4 \mathrm{GHz}$ and $5 \mathrm{GHz}$ has been kept at $20 \mathrm{MHz}$, while for $980 \mathrm{MHz}$, a bandwidth of $1 \mathrm{MHz}$ was assigned as lower bands cannot assume higher bandwidths, i.e., $980 \mathrm{MHz}$ can assume 


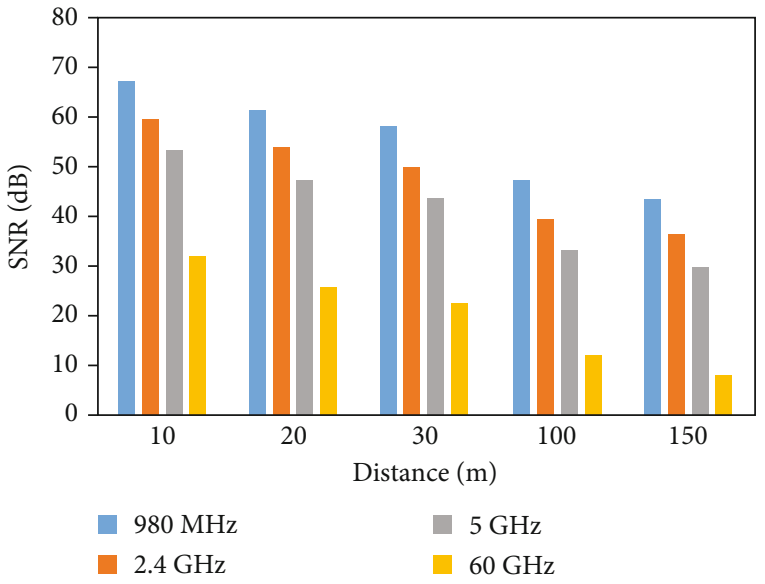

FIGURE 7: SNR with respect to distance.

a bandwidth of $1 \mathrm{MHz}$ only that is the limiting factor due to which lower bands have lower bitrate, hence lower throughput. Figure 8 shows that the optimal throughput for $980 \mathrm{MHz}, 2.4 \mathrm{GHz}$, and $5 \mathrm{GHz}$ is attained on the bitrate of $32 \mathrm{Mbps}$, $36 \mathrm{Mbps}$, and $40 \mathrm{Mbps}$, respectively. The graph tends to decrease when the optimal bitrate is less than bitrate, which leads to an increased bit error rate (BER). However, if the bitrate is less than optimal bitrate, then resources within channel capacity are underutilized. Apparently, it should have the lowest bitrate, and on the contrary, it has the highest bitrate. It all owes to the higher bandwidth that higher bands can assume.

6.3. Load Share Analysis. Based on the performance of different bands, using the proposed technique, the load share obtained for different bands is given in Figure 9. Generally, we know that the higher the frequency of the band, the higher will be the throughput, which we observed in Figure 8. Similarly, the result from analysis shows that $5 \mathrm{GHz}$ has the highest load share among all three bands, followed by $2.4 \mathrm{GHz}$ and finally $980 \mathrm{MHz} .5 \mathrm{GHz}$ shares more load, thereupon outperforming with the highest transmission rate and throughput. For $1000 \mathrm{MB}$ of total data, $5 \mathrm{GHz}$ can transmit a traffic load of about $500 \mathrm{MB}$, whereas $2.4 \mathrm{GHz}$ transmits a traffic load of $300 \mathrm{MB}$. $980 \mathrm{MHz}$, on the contrary, is having a load share of $200 \mathrm{MB}$.

6.4. End-to-End Delay Analysis. However, according to our proposed scheme in Figure 10, regardless of the load shares, the end-to-end delay of the three bands is the same; however, the curves coincide with each other, which incident upon one another appearing to be one curve only. This, in fact, shows that the three bands have an equal end-to-end delay. Delay being the same implies that packets arrive simultaneously. This, in return, ensures that there is no out-of-order arrival of packets which means packets are perfectly in order. Hence, there is no reordering delay involved in the transmitted data, which validates optimal load distribution in the given conditions.

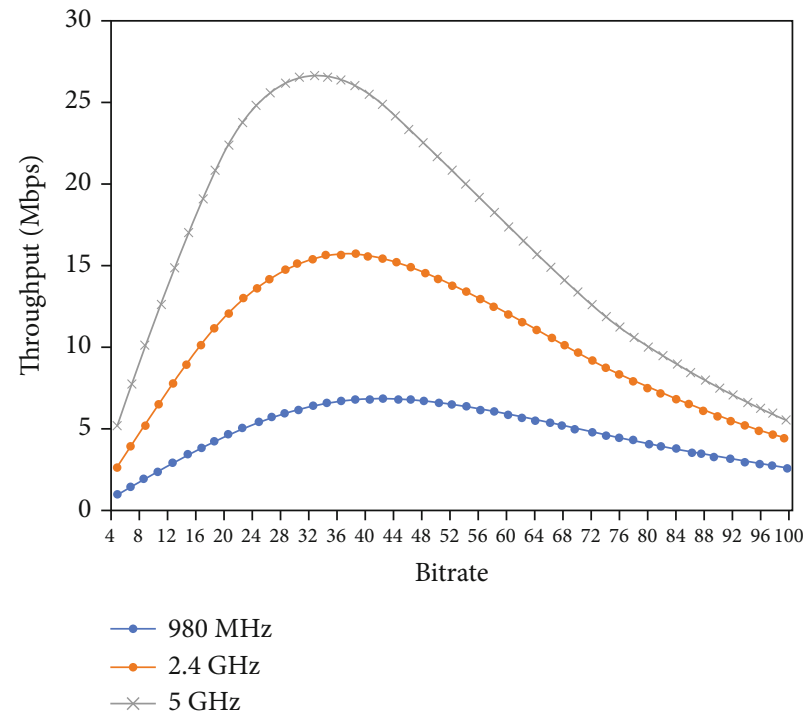

Figure 8: Transmission rate for different bands.

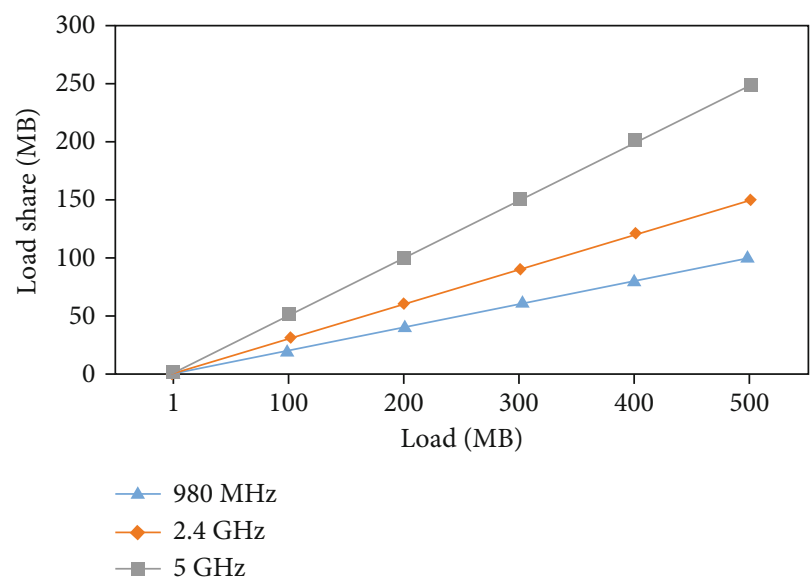

FIgURE 9: Load share distribution among the bands.

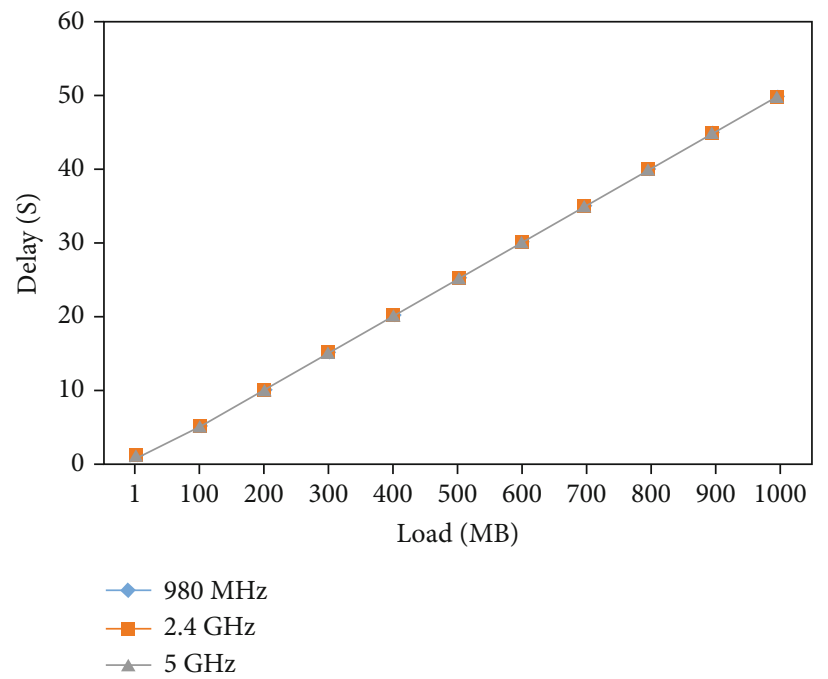

Figure 10: E2E delay for different bands. 
6.5. Change in the Load Share of $2.4 \mathrm{GHz}$ Bands. The effect of change in the traffic load of $2.4 \mathrm{GHz}$ is shown in Figure 11. The $x$-axis shows the increase in incoming load, which unveils change in the incoming load. Figure 11 shows the fairness distribution of the load corresponding to the performance of each band. Moreover, if additive nodes are incorporated at the relay node, this violates the delay constraint in MILP. In such a scenario, we need to switch the traffic load from $2.4 \mathrm{GHz}$ to $980 \mathrm{MHz}$ and $5 \mathrm{GHz}$, respectively, in order to maintain equal delay at the receiver's end. As the amount of incoming traffic load is increased by $2.4 \mathrm{GHz}$, the transmission rate is reduced ultimately, which caters to greater delay as compared to other bands. Thus, it is required to shift some of the traffic from the current band to the rest of the bands to ensure equal E2E delay. The switched traffic load is distributed with a proportional ratio according to each band's performance after being subtracted from the contemporary band. The traffic load, which increased initially, is now adjusted, and some amount of traffic from $2.4 \mathrm{GHz}$ is delegated to $980 \mathrm{MHz}$ and $5 \mathrm{GHz}$ bands. The new traffic load of $980 \mathrm{MHz}$ and $5 \mathrm{GHz}$ is also shown.

6.6. Change in the Traffic Load during a Random Walk of End-User. During the random walk, the location of the enduser changes continuously. As a result, channel conditions such as SNR, interference, and CSI change accordingly, which deteriorates in channel capacity, throughput, and delay of bands. Therefore, in Figure 12, we show the impacts of load share of different bands on the random walk of the end-user. The $x$-axis represents the distance covered by the end-user in terms of meters, and the $y$-axis shows the load share in MB. Whenever there is an increase in the load share of one band, there is an equal amount of decrease in other bands. The load share of $5 \mathrm{GHz}$ is decreased from 0 to 1 meter of distance, as depicted in Figure 12; alternately, this decrease is reflected with an increase in the load share of $2.4 \mathrm{GHz}$. This implies the same trend throughout the distance of 20 meters. The curve for change in the traffic load of $980 \mathrm{MHz}$ appears to be a straight line, which represents an invisible change in the load, initially due to a slight drop in the SNR of $980 \mathrm{MHz}$ with respect to distance, whereas $2.4 \mathrm{GHz}$ and $5 \mathrm{GHz}$ are more affected by the distance. Secondly, a meager amount of load is assumed by $980 \mathrm{MHz}$. Therefore, a minute change in such a meager amount of traffic load is very difficult to observe.

6.7. Traffic Switching Delay Analysis. In Figure 13, our proposed method compares the traffic switching delay performance with that of the Truncated Decode and Forward (TDF) scheme. TDF is a famous traffic switching scheme used in most of contemporary multiband systems [18, 19]. In the TDF scheme, whatever the traffic conditions may be at any node, the traffic is always switched from one to another band. On the contrary, traffic switching is subject to any change either in the condition of the channel within the band or in a load of the band. This means that the greater the number of relay nodes, the greater will be the traffic switching delay for TDF. Traffic switching delay is also the function of the number of relay nodes for our proposed

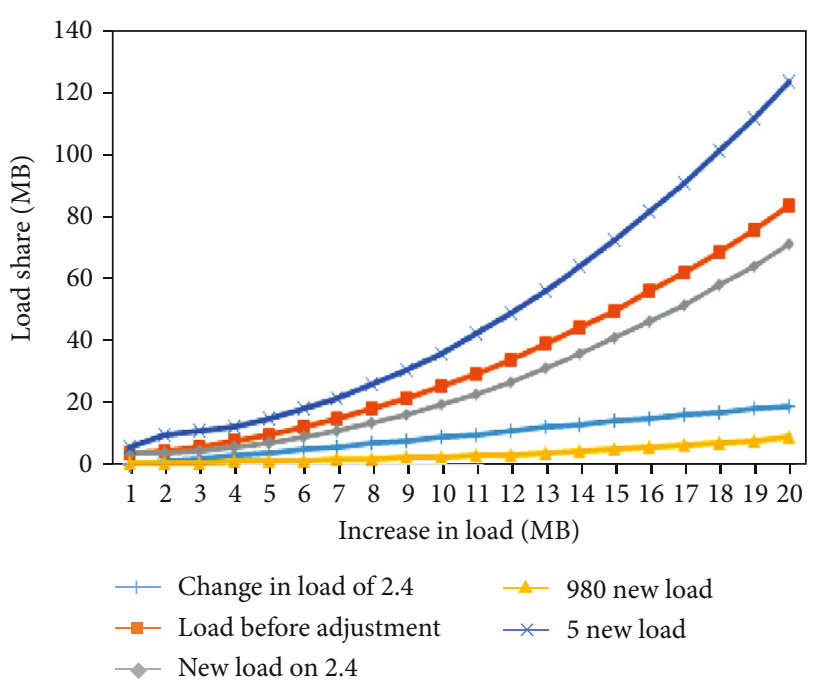

FIgURE 11: Change in the load share of $2.4 \mathrm{GHz}$ bands.

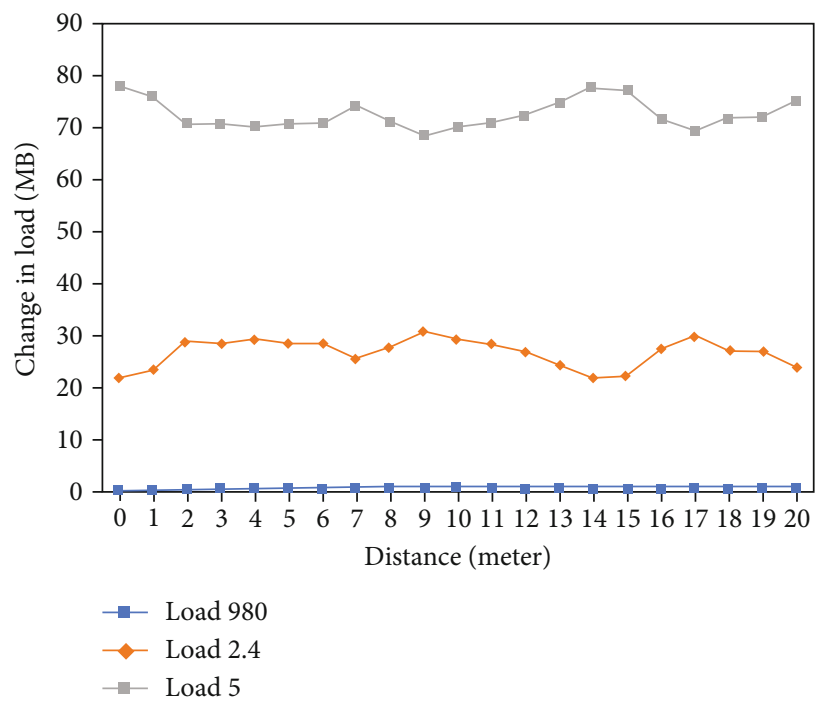

FIgUre 12: Change in load share for random walk.

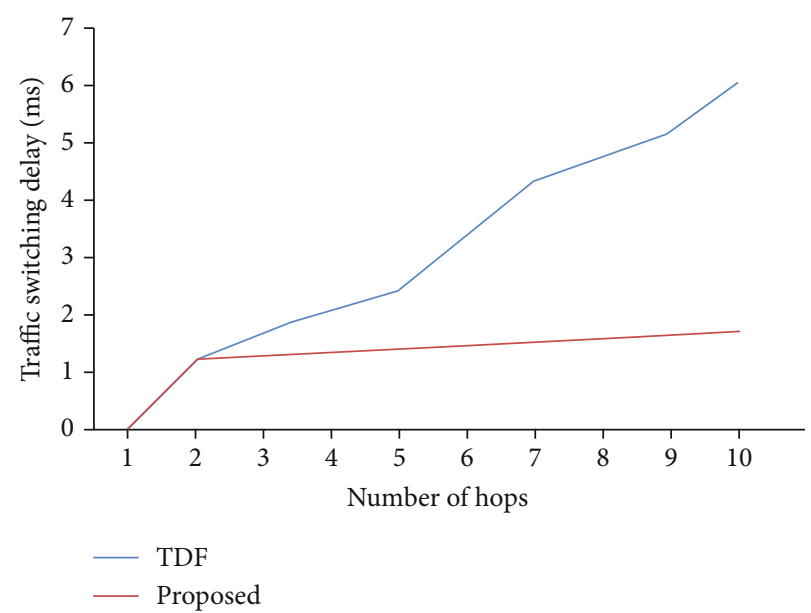

Figure 13: Traffic switching delay. 


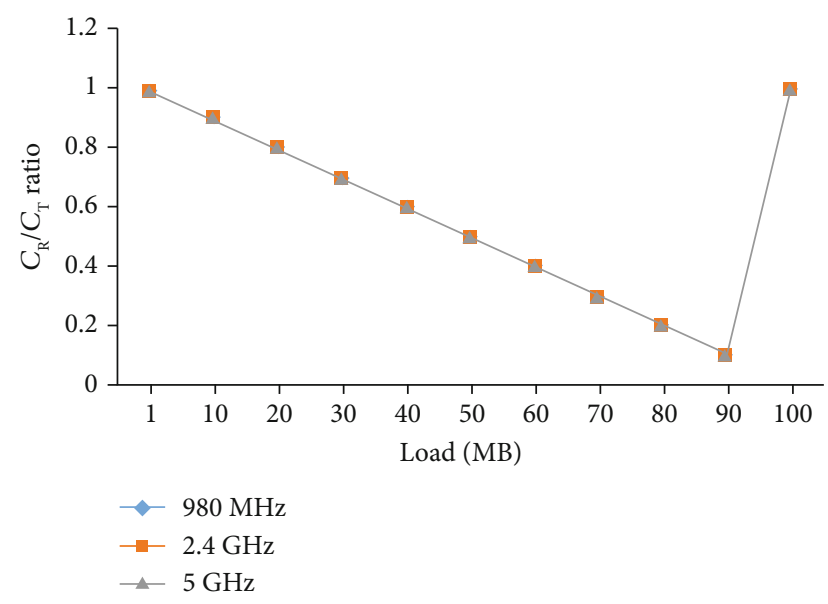

FIgURe 14: Residual capacity to total capacity ratio.

scheme as well. However, we switch only the needed amount of traffic. Figure 13 represents that in the case of TDF, switching delay is accelerating in a significant manner by increasing the hop counts accordingly, whereas the traffic switching delay of our proposed technique increases slightly by adding the relaying nodes. As shown in Figure 13, the total delay required for switching the traffic in TDF is approximately $6.5 \mathrm{~ms}$, while this is less than $1.7 \mathrm{~ms}$ in our proposed technique.

6.8. Capacity Utilization Analysis. Basically, the objective of residual capacity is bifold. We may improve throughput by minimizing the residual capacity of all the bands. On the other hand, the capacity utilization ratio must be the same by guaranteeing equal E2E delay among all the bands. In Figure 14, such ratio decreases firstly, which shows that the capacities are greater than the traffic load of the bands. The corresponding ratio intends to enhance as the traffic load surpasses the capacities. The three curves are coincident upon each other, giving the impression of one curve. This shows that the correspondent ratio of each band is equal by ignoring packet reordering delay.

6.9. Impact of End-Users on Load Share. As per IEEE 802.11 standard, $980 \mathrm{MHz}$ has only one channel, whereas the $2.4 \mathrm{GHz}$ band has 13 channels, out of which 3 are orthogonal, while the $5 \mathrm{GHz}$ band has a total of 54 channels among which 12 are orthogonal. This means that after 3 users if we add more users to the $2.4 \mathrm{GHz}$ band, the users will not have an orthogonal channel anymore. There will be interference among them, which will significantly reduce the throughput and end-to-end delay. The $5 \mathrm{GHz}$ band can tolerate about 12 users, while the $13^{\text {th }}$ user will impact the $5 \mathrm{GHz}$ band's performance. As earlier, all the users have orthogonal channels; they cannot affect each other in such conditions. Therefore, their end-to-end delay and throughput remain intact. This shows that the performance of $980 \mathrm{MHz}$ will be impacted if we have more than one user. Similarly, the performance of $2.4 \mathrm{GHz}$ will be affected by adding more than three users. Accordingly, their load will be shifted to $5 \mathrm{GHz}$ band, which is reflected in Figure 15. As the number of users

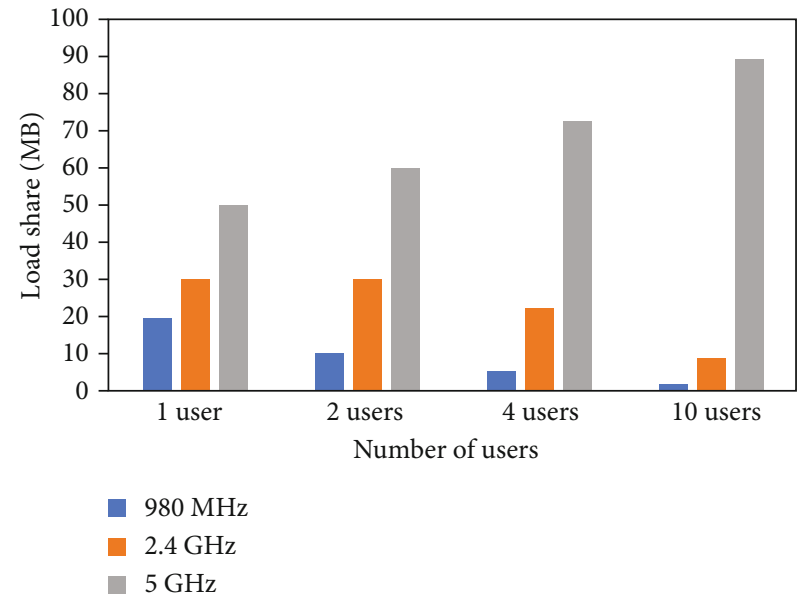

Figure 15: Impact of end-users on a load share.

increases, the load of $980 \mathrm{MHz}$ and $2.4 \mathrm{GHz}$ decreases gradually while $5 \mathrm{GHz}$ increases.

\section{Conclusion and Future Work}

In this study, we proposed simultaneous transmission over multihop WLAN systems. Multihop relay networks are deployed to restore the communication environment in the disastrous area rapidly. However, performance is deteriorated by adding relaying transmission. To cater to this situation, we commence simultaneous transmission with WLAN. Simultaneous multiband transmission in WLAN systems is believed to increase the coverage area without increasing the power. Channel conditions of different bands are different due to changes in their link conditions and transmission rates. This drastically degrades the performance of a network in terms of throughput and end-to-end delay. We formulate the mixed-integer linear programming (MILP) that is aimed at maximizing throughput and minimizing end-to-end delay. We propose a load distribution-based end-to-end traffic scheduling technique to improve system performance to address the problem. The simulation results show the effectiveness of our proposed method by maximizing throughput and minimizing end-to-end delay.

As an extension to this work, we plan to use game theory as an intelligent decision-making approach that provides effective resource allocation optimization problems. Machine learning can overcome the growing complexity of the network and, indeed, security threats. The column generation approach (CGA) is the decomposition technique for solving ILP problems into main problems and subproblem. CGA alternates between core problem and subproblem to find the optimal solution of the original ILP.

\section{Data Availability}

The data used to support the findings of this study are available from the corresponding author upon request. 


\section{Conflicts of Interest}

The authors declare that they have no conflicts of interest.

\section{Acknowledgments}

The authors are grateful to Taif University Researchers Supporting Project number TURSP-2020/215, Taif University, Taif, Saudi Arabia. This work was supported by the Grant System of University of Zilina No. 1/2020 (project no. 7962). This work is also supported by the Faculty of Computer Science and Information Technology, University of Malaya, under Postgraduate Research Grant (PG0352016A).

\section{References}

[1] Y. Jahir, M. Atiquzzaman, H. Refai, A. Paranjothi, and P. G. LoPresti, "Routing protocols and architecture for disaster area network: a survey," Ad Hoc Networks, vol. 82, pp. 1-14, 2019.

[2] S. A. Shah, D. Z. Seker, S. Hameed, and D. Draheim, "The rising role of big data analytics and IoT in disaster management: recent advances, taxonomy and prospects," IEEE Access, vol. 7, pp. 54595-54614, 2019.

[3] B. Shin and D. Lee, "An efficient local repair-based multiconstrained routing for congestion control in wireless mesh networks," Wireless Communications and Mobile Computing, vol. 2018, Article ID 2893494, 17 pages, 2018.

[4] T. Sakano, Z. M. Fadlullah, T. Ngo et al., "Disaster-resilient networking: a new vision based on movable and deployable resource units," IEEE Network, vol. 27, no. 4, pp. 40-46, 2013.

[5] Cisco Visual Networking Index, Forecast and Trends, 20172022, https://www.cisco.com/c/en/us/solutions/ serviceprovider/visual-networking-index-vni/index.htm.

[6] Erricsson, More Than 50 Billion Connected Devices, 2011, August 2019, https://www.akosrs.si/files/Telekomunikacije/ Digitalnaagenda/Internetniprotokol-Ipv6/More-than-50billion-connected-devices.pdf.

[7] M. Shafi, A. F. Molisch, P. J. Smith et al., "5G: a tutorial overview of standards, trials, challenges, deployment, and practice," IEEE Journal on Selected Areas in Communications, vol. 35, no. 6, pp. 1201-1221, 2017.

[8] K. H. Almotairi and W. S. Shen, "A distributed multi-channel MAC protocol for ad hoc wireless networks," IEEE Transaction on Mobile Computing, vol. 14, no. 1, pp. 1-13, 2014.

[9] L. Deek, E. Garcia-Villegas, E. Belding, S.-J. Lee, and K. Almeroth, "The impact of channel bonding on $802.11 \mathrm{n}$ network management," in Proceedings of the Seventh COnference on emerging Networking EXperiments and Technologies on CoNEXT '11, pp. 1-12, Tokyo, Japan, 2011.

[10] Z. J. Haas, "On the performance of a medium access control scheme for the reconfigurable wireless networks," in Advances in Wireless Communications. The International Series in Engineering and Computer Science, vol 435, J. M. Holtzman and M. Zorzi, Eds., pp. 269-284, Springer, Boston, MA, USA, 2002.

[11] M. Marvi, A. Aijaz, and M. Khurram, "Toward a unified framework for analysis of multi-RAT heterogeneous wireless networks," Wireless Communications and Mobile Computing, vol. 2019, Article ID 6918637, 19 pages, 2019.
[12] C.-M. Chao, T. Hsien-Chen, and H. Chao-Ying, "Load-aware channel hopping protocol design for mobile ad hoc networks," Wireless Networks, vol. 23, no. 1, pp. 89-101, 2017.

[13] H. Venkataraman, D. Gandhi, and V. Tomar, "Multi-hop multi-band intelligent relay-based architecture for LTEadvanced multi-hop wireless cellular networks," Wireless Personal Communications, vol. 75, no. 1, pp. 131-153, 2014.

[14] P. Kyasanur and N. H. Vaidya, "Routing and interface assignment in multi-channel multi-interface wireless networks," in IEEE Wireless Communications and Networking Conference, 2005, pp. 2051-2056, New Orleans, LA, USA, March 2005.

[15] L. Deek, E. G. Villegas, E. Belding, S. J. Lee, and K. Almeroth, "Intelligent channel bonding in 802.11n WLANs," IEEE Transaction on Mobile Computing, vol. 13, no. 6, pp. 12421255, 2014.

[16] F. Anwar, M. H. Masud, B. U. I. Khan, R. F. Olanrewaju, and S. A. Latif, "Analysis of packet reordering delay for bandwidth aggregation in heterogeneous wireless networks," IPASJ International Journal of Information Technology, vol. 6, no. 7, 2018.

[17] I. Marić, "Low latency communications," in 2013 Information Theory and Applications Workshop (ITA), pp. 1-6, San Diego, CA, USA, February 2013.

[18] C. Chereddi, P. Kyasanur, and N. H. Vaidya, "Design and implementation of a multi-channel multi-interface network," in Proceedings of the second international workshop on Multihop ad hoc networks: from theory to reality - REALMAN '06, pp. 23-30, Florence, Italy, May 2006.

[19] A. Hanyu, Y. Kawamoto, H. Nishiyama et al., "Adaptive frequency band and channel selection for simultaneous receiving and sending in multiband communication," IEEE Wireless Communications Letters, vol. 8, no. 2, pp. 460-463, 2019.

[20] Z. M. Fadlullah, Y. Kawamoto, H. Nishiyama et al., "Multi-hop wireless transmission in multiband WLAN systems: proposal and future perspective," IEEE Wireless Communications, vol. 26, no. 1, pp. 108-113, 2018.

[21] N. Egashira, K. Yano, S. Tsukamoto, J. Webber, and T. Kumagai, "Low latency relay processing scheme for WLAN systems employing multiband simultaneous transmission," in 2017 IEEE Wireless Communications and Networking Conference (WCNC), pp. 1-6, San Francisco, CA, USA, March 2017.

[22] A. Chaaban and A. Sezgin, "Multi-hop relaying: an end-to-end delay analysis," IEEE Transactions on Wireless Communications, vol. 15, no. 4, pp. 2552-2561, 2015.

[23] A. Chaaban and A. Sezgin, "When can a relay reduce end-toend communication delay?," in 2015 International Conference on Communications, Signal Processing, and their Applications (ICCSPA), pp. 1-6, Sharjah, United Arab Emirates, February 2015.

[24] K. Lee, A. Yener, and X. He, "Resource allocation for the multiband relay channel: a building block for hybrid wireless networks," EURASIP Journal on Wireless Communications and Networking, vol. 2010, no. 1, Article ID 792410, 2010.

[25] S. Sharma, Y. Shi, Y. Thomas Hou, H. D. Sherali, and S. Kompella, "Cooperative communications in multi-hop wireless networks: joint flow routing and relay node assignment," in 2010 Proceedings IEEE INFOCOM, pp. 1-9, San Diego, CA, USA, March 2010.

[26] X. Li, J. Luo, W. Wu, and M. Yang, "Joint AP coverage adjustment and user association optimization for load balancing in multi-rate WLANs," in 2017 IEEE 21st International 
Conference on Computer Supported Cooperative Work in Design (CSCWD), pp. 390-395, Wellington, New Zealand, April 2017.

[27] M. Li, S. Salinas, L. Pan, X. Huang, Y. Fang, and S. Glisic, "Optimal scheduling for multi-radio multi-channel multihop cognitive cellular networks," IEEE Transactions on Mobile Computing, vol. 14, no. 1, pp. 139-154, 2014.

[28] M. M. Fouda, H. Nishiyama, R. Miura, and N. Kato, "On efficient traffic distribution for disaster area communication using wireless mesh networks," Wireless Personal Communications, vol. 74, no. 4, pp. 1311-1327, 2014.

[29] R. Krishan and V. Laxmi, "IEEE 802.11 WLAN load balancing for network performance enhancement," Procedia Computer Science, vol. 57, pp. 493-499, 2015.

[30] S. Tani, K. Motoyoshi, H. Sano, A. Okamura, H. Nishiyama, and N. Kato, "Flexibility-enhanced HTS system for disaster management: responding to communication demand explosion in a disaster," IEEE Transactions on Emerging Topics in Computing, vol. 8, no. 1, pp. 159-167, 2020.

[31] D. Wang and C. H. Chan, "Multiband antenna for WiFi and WiGig communications," IEEE Antennas and Wireless Propagation Letters, vol. 15, pp. 309-312, 2015.

[32] J.-H. Lim, K. Naito, J.-H. Yun, and M. Gerla, "Exploiting overlapped bands for efficient broadcast in multichannel wireless networks," IEEE Transactions on Vehicular Technology, vol. 66, no. 5, pp. 4355-4370, 2016. 